\title{
New Catalysts and Receptors by Self-Assembly and Combinatorial Chemistry
}

\author{
Kay Severin* \\ Werner Prize Winner 2003
}

\begin{abstract}
New methods that allow the generation of transition metal-based catalysts and receptors are discussed. It is shown that heterobimetallic, halogeno-bridged complexes are well-suited for screening assays in combinatorial catalysis. Metal catalysts with a structurally defined second coordination sphere can be obtained by molecular imprinting with pseudo-substrate conjugates as exemplified by the substrate-, regio- and stereoselective reduction of ketones, catalyzed by immobilized ruthenium and rhodium complexes. Macrocyclic complexes, formed by self-assembly of organometallic complexes, are shown to act as highly specific receptors for small cations and anions.
\end{abstract}

Keywords: Catalysis · Combinatorial Chemistry $\cdot$ Host-Guest Chemistry $\cdot$ Metal Complexes $\cdot$ Self-Assembly

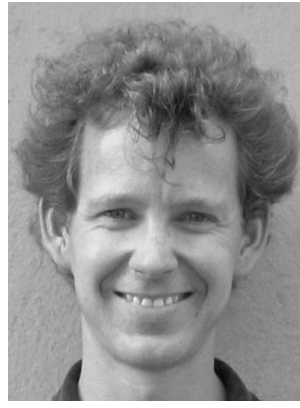

Kay Severin was born in Germany in 1967. He obtained his Ph.D. in 1995 with a thesis on bioorganometallic chemistry, carried out in the group of Prof. W. Beck, University of Munich. Subsequently, he joined the group of Prof. M.R. Ghadiri, Scripps Research Institute, to study selfreplicating peptides, autocatalytic networks, and an artificial peptide ligase. In 1997, he started independent research projects ('Habilitation') at the Department of Chemistry, University of Munich. After a visiting professorship at the University of Vienna, he joined the Institute of Inorganic Chemistry at the University of Lausanne in August 2001. Since October 2001 he is assistant professor at the Institute of Chemical Sciences and Engineering at the Swiss Federal Institute of Technology, Lausanne (EPFL). His work has been honored by several awards: Bayerischer Habilitations Förderpreis (1997), ADUC award of the year for outstanding work during the Habilitation (2001), award of the Karl-Ziegler foundation (2001), Arnold Sommerfeld award of the Bavarian Academy of Science (2001) and the Heinz Maier-Leibnitz award of the DFG (2001).

\footnotetext{
${ }^{*}$ Correspondence: Prof. K. Severin

Institut des Sciences et Ingénierie Chimique

École Polytechnique Fédérale de Lausanne

$\mathrm{CH}-1015$ Lausanne

Tel.: +41216939302

Fax: +41216939305

E-Mail: kay.severin@epfl.ch

http://isic.epfl.ch/severin e.htm
}

The following article provides a brief overview about the different research projects that are currently being pursued in my laboratory at the École Polytechnique Fédérale de Lausanne. It is based on a talk given on the occasion of the Fall Meeting 2003 of the Swiss Chemical Society.

\section{Combinatorial Catalysis with Bimetallic Complexes}

Combinatorial methods are increasingly being used for the discovery and the optimization of new catalysts. In the field of homogeneous catalysis, most efforts have focused on mononuclear complexes using combinatorial libraries of ligands [1]. It would be interesting, however, to include polynuclear complexes. First of all, because the presence of two or more metal ions would allow the diversity of the library to be increased further and secondly, because of the potentially superior performance of polynuclear catalysts as compared to their mononuclear counterparts. A prerequisite for a fast screening assay is the availability of a library of potential catalyst precursors. For polynuclear complexes one is thus faced with the problem to synthesize a structurally diverse set of compounds on a reasonable time scale.

Over the last years, we have developed very efficient and versatile methods, which allow the synthesis of bimetallic complex- es, in which two different metal fragments are connected by either two [2-5] or three [6-8] halogeno-bridges [9]. Some of the complexes, which are suited for the synthesis of asymmetric complexes with three halogeno-bridges are shown in Scheme 1. Various transition metals such as ruthenium, rhodium, iridium, rhenium, molybdenum or tungsten can be incorporated and a variety of different ligands can be employed. Importantly, it is possible to combine every complex of type A with every complex of type B. As a consequence, the structural diversity that is accessible is very large, even for the small subset of starting materials that is shown in Scheme 1. For applications in the field of combinatorial catalysis, it is also of importance that bimetallic, halogeno-bridged complexes can be synthesized in situ without prior purification. This facilitates the screening assays to a considerable extent. One should note, however, that this class of compounds is less suited for catalytic reactions with substrates which represent strong donor ligands, because cleavage of the halogenobridge can occur.

Recently, we have reported a first application in homogeneous catalysis [10]. A library of 66 catalyst precursors was generated in situ in a highly parallel fashion by mixing chloro-bridged complexes of $\mathrm{Rh}^{\mathrm{I}}$, $\mathrm{Rh}^{\mathrm{III}}, \mathrm{Ir}^{\mathrm{I}}, \mathrm{Ir}^{\mathrm{III}}, \mathrm{Pd}^{\mathrm{II}} \mathrm{Ru}^{\mathrm{II}}, \mathrm{Ru}^{\mathrm{III}}, \mathrm{Ru}^{\mathrm{IV}}$, and $\mathrm{Pt}^{\mathrm{II}}$ with the three different $\mathrm{Ru}^{\mathrm{II}}$-phosphine complexes. As a benchmark reaction for 


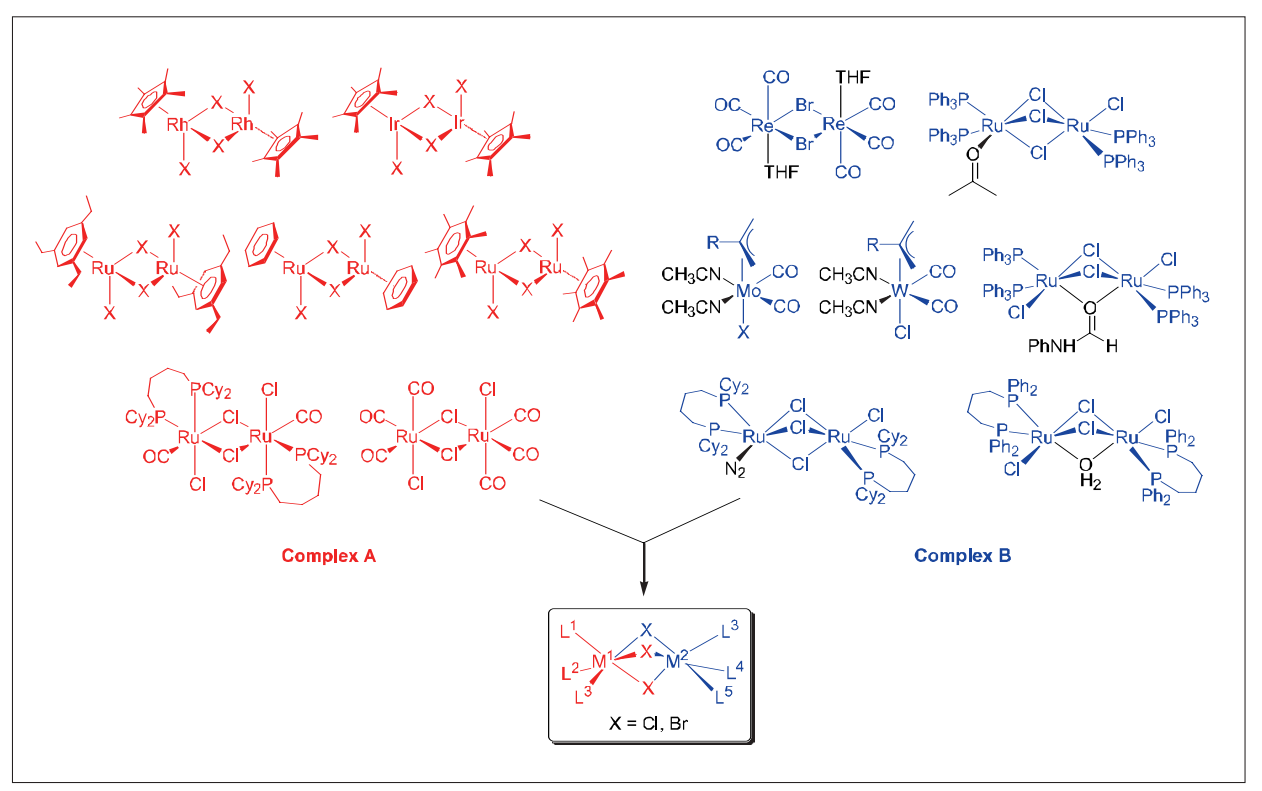

Scheme 1. Synthesis of bimetallic complexes in which two different metal fragments are connected by three halogeno-bridges. Every complex of type A can be combined with every complex of type B. Only a subset of potential starting materials is shown.

atom transfer radical additions (ATRA) we have used the addition of $\mathrm{CCl}_{4}$ to styrene. Based on this assay, we have been able to identify two heterometallic, chloro-bridged Rh-Ru complexes which show an exceptionally high activity (Fig. 1). With a maxi-

mum TON of 4500 and an initial TOF of $1200 \mathrm{~h}^{-1}$, they rank among the best ATRA catalysts described so far. Furthermore, these new catalysts tolerate the use of water-containing solvents and give excellent yields for 'difficult' substrates such as

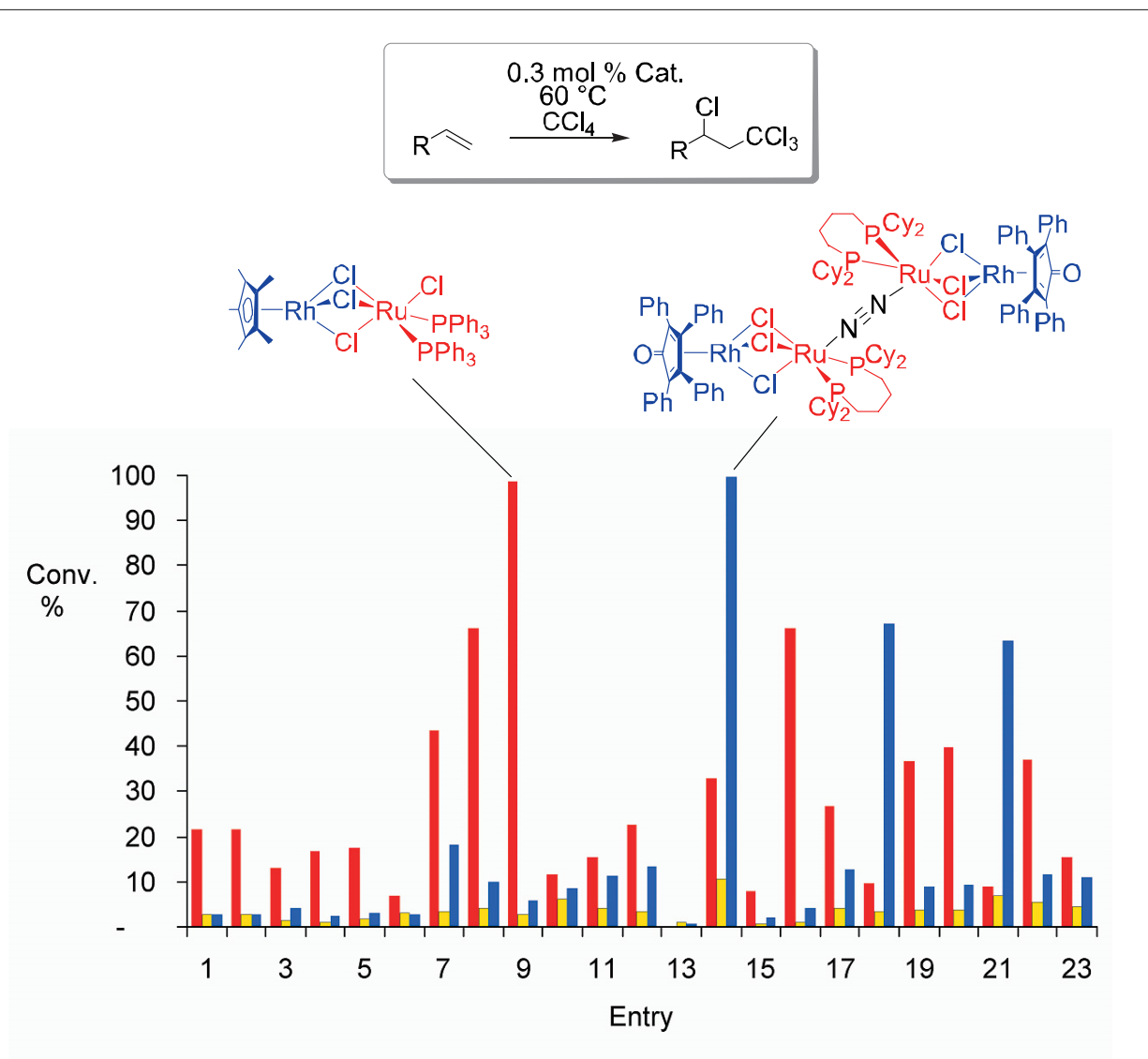

Fig. 1. Two chloro-bridged Rh-Ru complexes are identified as highly active catalysts for the atom transfer radical addition of $\mathrm{CCl}_{4}$ to olefins using a fast screening assay with in situ generated polynuclear complexes octene. These results clearly demonstrate the potential of mixed halogeno-bridged complexes in homogeneous catalysis. Other reactions such as atom transfer radical polymerizations are currently being investigated in our laboratory.

\section{Biomimetic Catalysis by Molecular Imprinting}

The fact that bimetallic complexes are potentially superior catalysts is a lesson that chemists have learned many years ago by studying metalloenzymes. Another feature of metalloenzymes is that the selectivity is often controlled by a structurally defined second coordination sphere of the metal complex. For the design of synthetic catalysts, the second coordination sphere is generally neglected, mostly due to a lack of suited methods to make such complexes. Nevertheless, the implementation of a structurally defined microenvironment in synthetic organometallic catalysts would be of interest for several applications. Due to specific molecular recognition between substrate and catalyst an enhanced regio-, stereo-, and substrate-selectivity should be possible. In order to reach this goal we use a simple and flexible method that is based on self-assembly, called molecular imprinting (MI) [11]. The strategy how this technique can be employed to control the microenvironment of a transition metal catalyst is outlined in Scheme 2. A catalytically active metal complex having one (or more) polymerizable side chain(s) is coordinated to a pseudo-substrate (step a). In terms of size and functionality this pseudo-substrate should be similar to the real substrate used in the catalytic transformation. Ideally, the resulting complex should mimic the transition state of the catalytic transformation. The catalyst-pseudo-substrate conjugate is subsequently co-polymerized with a large excess of a crosslinking monomer (e.g. ethylene glycol dimethacrylate) in the presence of a porogen following the standard protocol of MI (step b). Selective removal of the pseudo-substrate from the resulting polymer gives an immobilized catalyst with a specific cavity in proximity to the catalytically active metal center ( tively, the metal ion is also removed from the polymer (step d). The catalyst is then assembled in a final step on the support (step e). The advantage of the latter method is that it is possible to switch metal ions (e.g. a $\mathrm{Cu}$ complex for imprinting and a $\mathrm{Rh}$ complex for catalysis). It is important to point out that the highly crosslinked polymers generated in such a procedure are ideally suited for immobilized catalysts. Due to their high surface area - typically between 100 and $500 \mathrm{~m}^{2} / \mathrm{g}$ are observed - the accessibility of the catalyst within the polymer is 


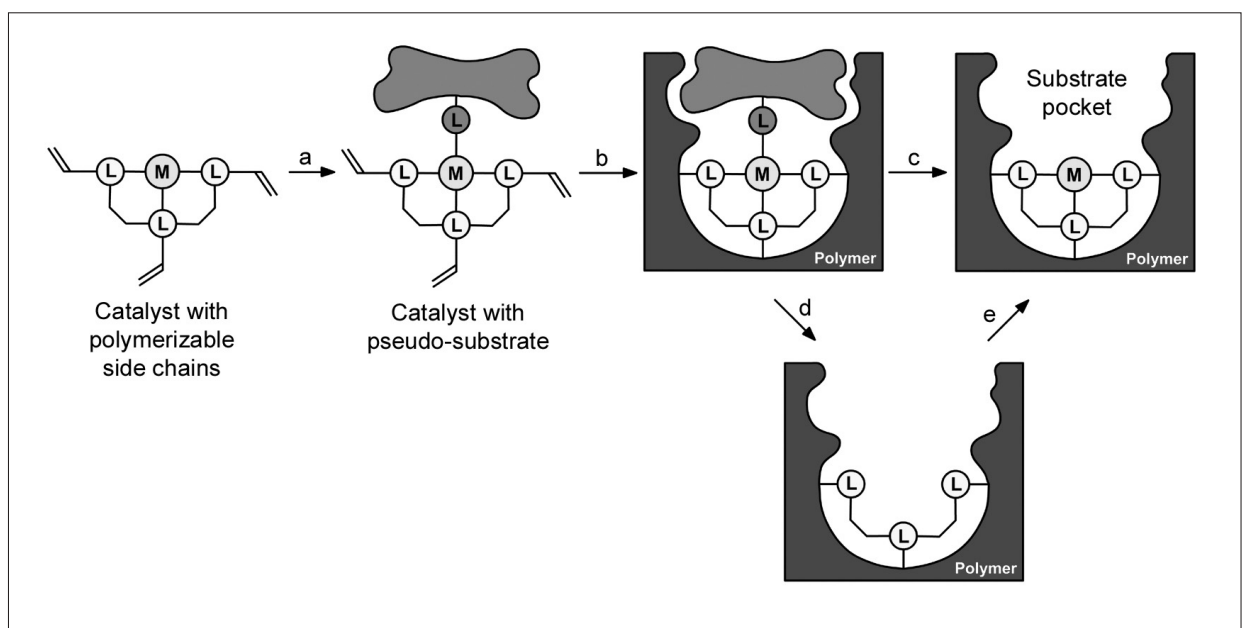

Scheme 2. Basic concept to generate an immobilized catalyst with a well-defined second coordination sphere ('substrate pocket') by molecular imprinting

very good. Furthermore, the permanent pore structure allows the use of a large variety of solvents for catalysis, even water.

We have employed the methodology outlined in Scheme 2 for different transition metal catalysts, in particular for transfer hydrogenations catalyzed by organometallic ruthenium and rhodium complexes [12]. If immobilized catalysts with and without substrate pocket are compared, one can generally observe an increased activity for the imprinted catalysts by up to one order of magnitude. But more importantly, it is possible to modulate the substrate- [13][14], stereo- [15] and regioselectivity [14] in a controlled fashion. Apart from an increased selectivity, imprinted metal catalysts display two additional advantages: a) Destructive intermolecular reactions are diminished by site isolation in the polymeric matrix. As a result, the stability of the catalyst may be enhanced [16]. b) The polymers display the classical advantages of heterogeneous catalysts such as easy recovery by filtration, which may be of interest for applications in organic synthesis.

\section{Self-Assembled Receptors for Small Cations and Anions}

Compared to specific catalysts, the structural requirements for selective ionophores are slightly different. Whereas for catalysts with a substrate pocket a certain flexibility in the binding site can be tolerated and may even be needed, a good ionophore with a high selectivity needs to be very rigid and preorganized. This is nicely illustrated by the 'spherands' developed by Cram [17]. Here, the oxygen donoratoms are ideally preorganized to bind small alkali metal ions and a remarkable binding constant combined with an excel- lent selectivity is observed. But the drawback - from a synthetic point of view - is obvious: it's very hard to make these molecules. A very attractive alternative for the construction of macrocyclic ionophores is the utilization of transition metal based self-assembly processes. These reactions often give rather rigid structures in a single step and in good yields. First reports about so-called metallacrown complexes were published by Pecoraro et al. at the beginning of the 90s [18] but in recent years, many other research groups have contributed to this area (for some selected references see [19-22]).

The class of compounds that we are focusing on are macrocycles which are obtained by reaction of tridentate ligands with organometallic complexes of (arene) $\mathrm{Ru}^{\mathrm{II}}$ [23-32], Cp*Ir III [31-33], Cp*Rh ${ }^{\text {III }}$ [24][30] and (CO) ${ }_{3} \operatorname{Re}^{\mathrm{I}}$ [34] (Fig. 2). All these metal fragments possess three available facial coordination sites. If sufficiently rigid ligands are employed, tri- or tetranuclear complexes can be formed in excellent yields (Fig. 3).

Several of these complexes were found to display an interesting host-guest chemistry [35][36]. Trinuclear complexes build with 2,3-dihydroxypyridine ligands, for example, represent analogues of 12-crown-3. Similar to their organic counterparts they can bind lithium and sodium ions, although with much higher affinity and selectivity [23-25]. This was used to develop a specific chemosensor for $\mathrm{Li}^{+}$[28] and to stabilize the unusual molecular forms of $\mathrm{LiF}$ [31] and $\mathrm{Na}_{2} \mathrm{SiF}_{6}$ [26]. If 2,3-dihydroxypyridine ligands with amino-substituents are employed, it is possible to generate trinuclear macrocycles, which are soluble in water at neutral $\mathrm{pH}$. The corresponding (cymene) $\mathrm{Ru}$ complex is among the best and selective receptors for lithium ions in water which have been described so far [29]. Due to the pharmacological interest of lithium salts, biomedical applications can be envisioned [37].

In an extension of this work, we have been able to construct a specific receptor for the fluoride anion [33]. The basic concept is schematically shown in Fig. 4. A lithium ion, which serves as a fluoride-binding site, is coordinated inside a trimeric Cp*Ir complex. The accessibility of the $\mathrm{Li}^{+}$center is limited by the steric requirements of the $\pi$ ligand. Since the radius of the fluoride ion is significantly smaller than that of most other anions and since the hard Lewis acid $\mathrm{Li}^{+}$has a high intrinsic affinity for the hard Lewis base $\mathrm{F}^{-}$, a highly specific receptor is obtained. An important feature of this receptor is the fact that the binding of fluoride can be transduced into an electrochemical signal. The system can thus be used as a specific chemosensor.

Currently, we are investigating the dynamic behavior of these macrocyclic ionophores. It was shown that in polar solvents, the complexes undergo scrambling reactions. If a trimeric $\mathrm{Cp} * \mathrm{Rh}$ complex is mixed with a trimeric (arene) Ru complex, for example, heterometallic Rh-Rh-Ru and $\mathrm{Ru}-\mathrm{Ru}-\mathrm{Rh}$ macrocycles are obtained rapidly. This offers the opportunity to generate dynamic combinatorial libraries (DCL) of receptors [38]. A DCL represents a chemical network that is able to adapt to the environment. If a target molecule is added to such a system, it will re-equilibrate until the new thermodynamic minimum is established. Several groups have shown that the re-equilibration can lead to the amplification of molecules with high affinity to the target [38]. This amplification can be used to discover new receptors or - depending on the target molecule - new catalysts and substrates.

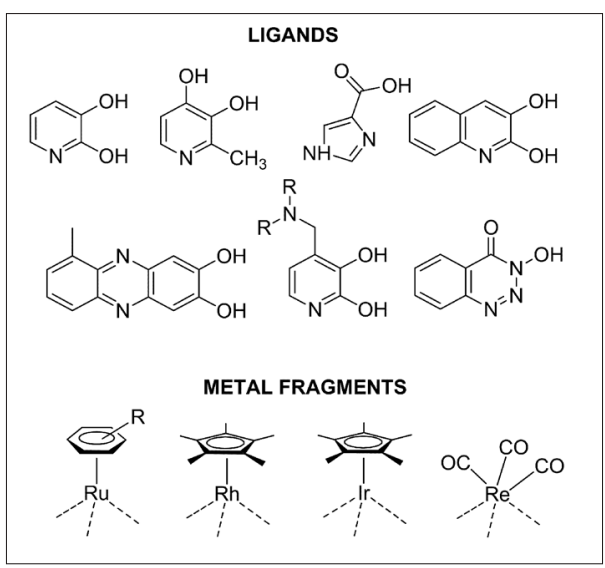

Fig. 2. The combination of tridentate ligands with organometallic complexes having three available binding sites can lead to the formation of macrocyclic complexes with interesting host-guest properties 


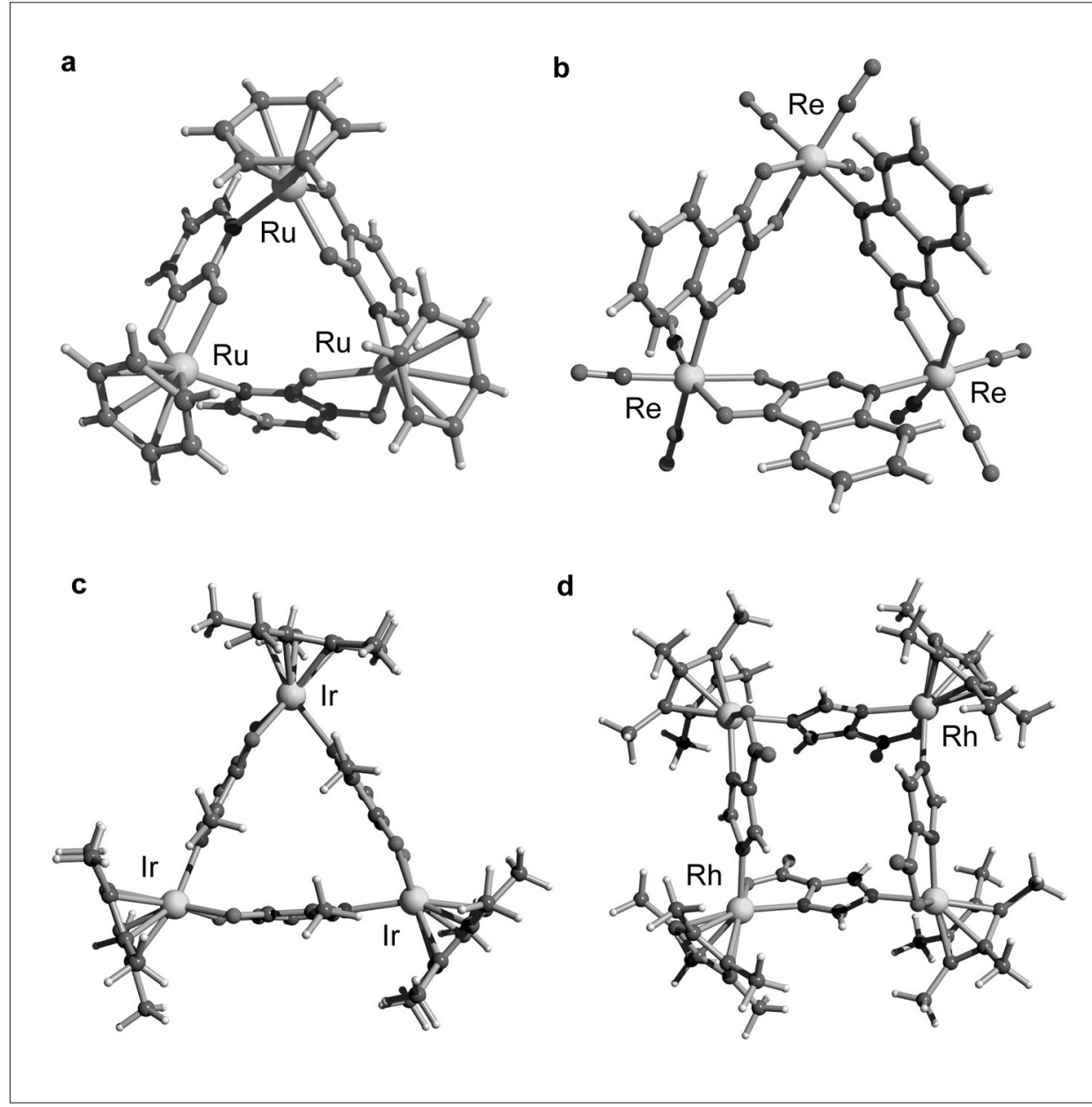

Fig. 3. Molecular structures of organometallic macrocycles containing a) (arene)Ru-, b) (CO) ${ }_{3} \mathrm{Re}-, \mathrm{c}$ ) $\mathrm{Cp}^{*} \mathrm{Ir}-$ or d) $\mathrm{Cp}^{*} \mathrm{Rh}$ fragments. The (arene)Ru complex can act as a highly selective receptor for lithium and sodium salts whereas the $(\mathrm{CO})_{3}$ Re complex can be used to stabilize an unusual silver-arene complex.
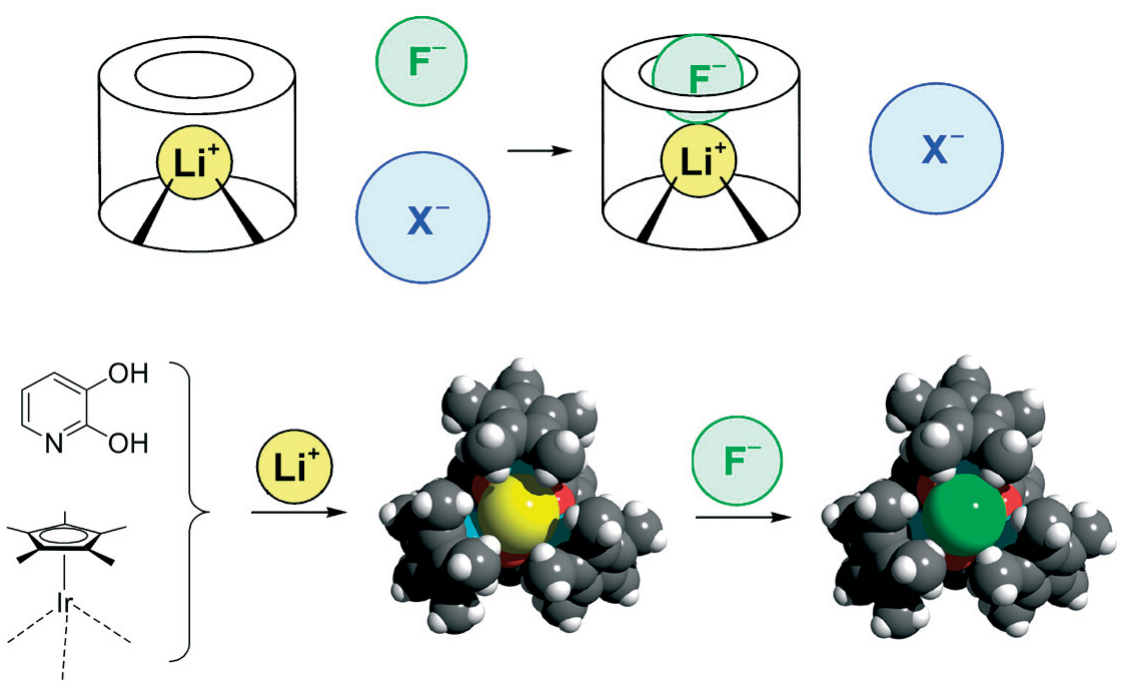

Fig. 4. A lithium ion, coordinated to a receptor comprised of three $\mathrm{Cp}^{*}$ Ir complexes with bridging dihydroxypyridine ligands, acts as a highly specific binding site for the fluoride anion.
In a combined theoretical and experimental study, we have recently identified some previously unnoticed limitations of the DCL approach [39][40]. But these limitations are only valid for certain boundary conditions, as numerical simulations have shown [40]. Based on this knowledge, we are now using DCLs of metallamacrocycles for the detection of new high-affinity receptors.

\section{Acknowledgements}

The work described in this overview was supported by the Swiss National Science Foundation and by the DFG. I am grateful to all coworkers who have contributed to this work.

Received: January 22, 2004

[1] B. Jandeleit, D.J. Schaefer, T.S. Powers, H.W. Turner, W.H. Weinberg, Angew. Chem. Int. Ed. 1999, 4, 2495-2532.

[2] K. Polborn, K. Severin, Eur. J. Inorg. Chem. 1998, 1187-1192.

[3] K. Polborn, K. Severin, J. Chem. Soc., Dalton Trans. 1999, 759-764.

[4] M. Öhm, A. Schulz, K. Severin, Eur. J. Inorg. Chem. 2000, 2623-2629.

[5] E. Kessenich, A. Schulz, K. Severin, J. Raman Spectr. 2001, 32, 241-250.

[6] A.C. da Silva, H. Piotrowski, P. Mayer, K. Polborn, K. Severin, J. Chem. Soc., Dalton Trans. 2000, 2960-2963.

[7] A.C. da Silva, H. Piotrowski, P. Mayer, K. Polborn, K. Severin, Eur. J. Inorg. Chem. 2001, 685-691.

[8] S. Gauthier, L. Quebatte, R. Scopelliti, K. Severin, Chem. Eur. J. 2004, in press.

[9] For an overview see: K. Severin, Chem. Eur. J. 2002, 8, 1514-1518.

[10] L. Quebatte, R. Scopelliti, K. Severin, Angew. Chem. Int. Ed. 2004, 43, 1520 1524.

[11] 'Molecularly Imprinted Polymers. ManMade Mimics of Antibodies and their Applications in Analytical Chemistry', Ed. B. Sellergren, Elsevier, New York, 2001.

[12] K. Severin, Curr. Opin. Chem. Biol. 2000, 4, 710-714.

[13] K. Polborn, K. Severin, Chem. Commun. 1999, 2481-2482.

[14] K. Polborn, K. Severin, Chem. Eur. J. 2000, 6, 4604-4611.

[15] K. Polborn, K. Severin, Eur. J. Inorg. Chem. 2000, 1687-1692.

[16] O. Nestler, K. Severin, Org. Lett. 2001, 3, 3907-3909.

[17] D.J. Cram, Science 1983, 219, 1177-1183.

[18] V.L. Pecoraro, A.J. Stemmler, B.R. Gibney, J.J. Bodwin, H. Wang, J.W. Kampf, A. Barwinski, Prog. Inorg. Chem., Ed. K. Karlin; Pergamon Press, New York, 1997; Vol. 45, p. 83-177.

[19] S.-X., Liu, S. Lin, B.-Z. Lin, C.-C. Lin, J.Q. Huang, Angew. Chem. Int. Ed. 2001, 40, 1084-1087.

[20] R.W. Saalfrank, I. Bernt, F. Hample, Angew. Chem. Int. Ed. 2001, 40, 1700-1703. 
[21] H. Rauter, E.C. Hillgreis, A. Erxleben, B. Lippert, J. Am. Chem. Soc. 1994, 116, 616-624.

[22] A. Caneschi, A. Cornia, S.J. Lippard, Angew. Chem. Int. Ed. 1995, 34, 467-469.

[23] H. Piotrowski, K. Polborn, G. Hilt, K. Severin, J. Am. Chem. Soc. 2001, 123, 2699-2700.

[24] H. Piotrowski, G. Hilt, A. Schulz, P. Mayer, K. Polborn, K. Severin, Chem. Eur. J. 2001, 7, 3197-3208.

[25] M.-L. Lehaire, A. Schulz, R. Scopelliti, K. Severin, Inorg. Chem. 2003, 42, 3576-3581.

[26] M.-L. Lehaire, R. Scopelliti, K. Severin, Chem. Commun. 2002, 2766-2767.
[28] H. Piotrowski, K. Severin, Proc. Natl. Acad. Sci. USA 2002, 99, 4997-5000.

[29] Z. Grote, M.-L. Lehaire, R. Scopelliti, K. Severin, J. Am. Chem. Soc. 2003, 125, 13638-13639.

[30] M.-L. Lehaire, L. Herdeis, R. Scopelliti, K. Polborn, P. Mayer, K. Severin, Inorg. Chem. 2004, in press.

[31] M.-L. Lehaire, R. Scopelliti, K. Severin, Inorg. Chem. 2002, 41, 5466-5474.

[32] T. Habereder, M. Warchhold, H. Nöth, K. Severin, Angew. Chem. Int. Ed. 1999, 38, 3225-3228.

[33] M.-L. Lehaire, R. Scopelliti, H. Piotrowski, K. Severin, Angew. Chem. Int. Ed. 2002, 41, 1419-1422.
[34] T. Brasey, A. Buryak, R. Scopelliti, K. Severin, Eur. J. Inorg. Chem. 2004, 964-967.

[35] K. Severin, Coord. Chem. Rev. 2003, 245 , 3-10.

[36] M.-L. Lehaire, K. Severin, Chimia 2003 , 57, 190-192.

[37] G.D. Christian, Sensors, 2002, 2, 432-435.

[38] S. Otto, R.L.E. Furlan, J.K.M. Sanders, Curr. Opin. Chem. Biol. 2002, 6, 321-327.

[39] Z. Grote, R. Scopelliti, K. Severin, Angew. Chem. Int. Ed. 2003, 42, 3821-3825.

[40] K. Severin, Chem. Eur. J. 2004, in press. 